\title{
Microscopic second harmonic generation (SHG) from tilt-placed collagen fibrils
}

\author{
Ying Chang ${ }^{1}$, Huajiang Wei ${ }^{1}$, Ying Jin ${ }^{1}$, Hanping Liu ${ }^{1}$ and Xiaoyuan Deng ${ }^{1,2}$ \\ ${ }^{1}$ MOE Key Laboratory of Laser Life Science, South China Normal University, Guangzhou 510631, China \\ ${ }^{2}$ Research Resources Center, South China Normal University, Guangzhou Higher Education Mega Center 510006, China
}

\begin{abstract}
We report a theoretical study of second-harmonic generation (SHG) from the collagen fibrils which have a tilt angle $\Phi$ between their axes with the plane of the polarization angle $\alpha$ of the linearly polarized focused light. The effects of $\Phi$ as well as $\alpha$ on SHG emission have been investigated. Our results show that the total strength of SHG electrical field $\left(\mathrm{E}_{2 \omega}\right)$ and power $\left(\mathrm{P}_{2 \omega}\right)$ reach their maximal values as $\Phi$ locates at a certain angle, here it is around $27^{\circ}$ under all demonstrated $\alpha$. When $\Phi<27^{\circ}, \mathrm{E}_{2 \omega}$ and $\mathrm{P}_{2 \omega}$ gradually increase to their maximal value, while when $\Phi>27^{\circ}$, they quickly drops. Specially, $\mathrm{E}_{2 \omega}$ and $\mathrm{P}_{2 \omega}$ are almost undetectable when $\Phi \geq 45^{\circ}$. Also, $\Phi$ influences the distribution pattern of $\mathrm{E}_{2 \omega}, \mathrm{P}_{2 \omega}$ and their parallel $\left(\mathrm{E}_{2 \omega, \mathrm{p}}, \mathrm{P}_{2 \omega, \mathrm{p}}\right)$ and perpendicular $\left(\mathrm{E}_{2 \omega, \mathrm{s}}, \mathrm{P}_{2 \omega, \mathrm{s}}\right)$ components, such as the number of emission lobes and shape. $\alpha$ has unevenly impact on $E_{2 \omega}$ and $P_{2 \omega}$, the far deviation of $\alpha$ from $\mathbf{x}$ axis induce much great decrease of $\mathrm{E}_{2 \omega}$ and $\mathrm{P}_{2 \omega} \cdot \alpha=45^{\circ}$ has special influence on the distribution pattern of $\mathrm{E}_{2 \omega, s}, \mathrm{E}_{2 \omega}, \mathrm{P}_{2 \omega, \mathrm{s}}$ as well as $\mathrm{P}_{2 \omega}$ compared to those of $\alpha=0^{\circ}$ and $90^{\circ}$.
\end{abstract}

Key words: Linearly polarization - Fibrils orientation - Strength of SHG electric field and power

\section{Introduction}

The process of second-harmonic generation (SHG), which occurs by the scattering of photons in the material with the non-centrosymmetric structural feature, thus unlike the absorption process as the fluorescence generation, there is no energy loss in SHG during its excitation to emission. Also, SHG is the intrinsic signal; no additional fluorescent staining is required. SHG thus has unique advantages such as no photobleaching, no photoxicity caused in the specimen (Brown et al. 2003; Zipfel et al. 2003; Helmchen et al. 2006).

Taking this well-known optical effect, microscopic SHG imaging has been exploited to be a powerful tool for visualization of biological systems. Fibrillous collagen type I, one of the strongest SHG producers of biological specimen, has been widely investigated for imaging in biological tissue experimentally and theoretically (Williams et al. 2005; Plotnikov et al. 2006; Yew et al. 2007). Fibrillous collagen type I, as its polymerization or degradation evolves in par-

Correspondence to: Xiaoyuan Deng, MOE Key Laboratory of Laser Life Science, South China Normal University, Guangzhou 510631, China

E-mail: xiaoyuandeng@gmail.com allel with the evolution of the cancerous lesions, has been experimentally investigated through microscopic SHG imaging for cancer diagnosis by identifying its content, spatial distribution and fiber organization (Hompland et al. 2008; Lamonier et al. 2010). Also, as SHG originates from the laserinduced second-order polarization, this signal is sensitive to the incoming excitation polarization and to the SHG active structures' architectures. Taking advantage of the effects of excitation polarization on tissue SHG, the architecture of their contributors, such as collagen, myosin as well as microtubule can be characterized. The estimated effective angles $\theta_{\mathrm{e}}$, which is corresponding to the most probable orientation of the active molecules, in neurons, in collagen (correlated to the helical pitch angle of one polypeptide chain of the collagen triple-helix) and in myosin (correlated to the $\alpha$-helix of the myosin's coiled coil) have been measured (Tiaho et al. 2007; Kwan et al. 2008; Psilodimitrakopoulos et al. 2009, 2010). Meanwhile, a series of theories dealing with SHG excited by linearly polarized focused beam from collagen fibrils have been explored (Stoller et al. 2002; Stoller et al. 2003; Yan et al. 2007). However, so far, the theoretical studies with regard to SHG from collagen fibrils have an assumption that the collagen fibrils locate in the same plane of the polarization direction of incident light (Yew and 


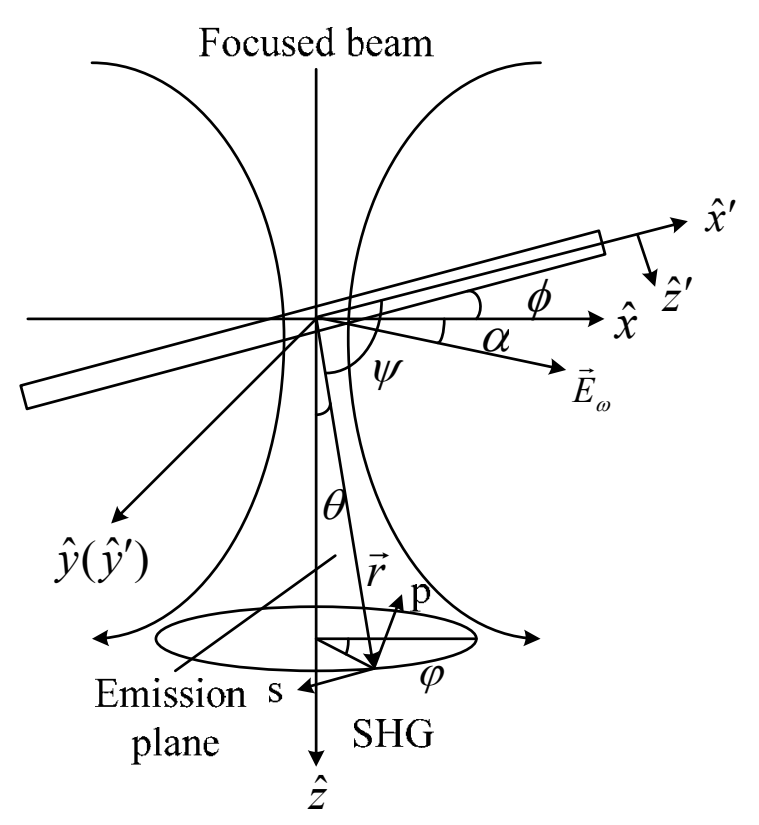

Figure 1. Schematic diagram defining SHG emission from collagen fibrils under linearly polarized focused beam. Collagen fibrils are assumed in $\mathbf{x}-\mathbf{z}$ plane with the axis of $\mathbf{x}$ '. It deviates from the $\mathbf{x}$ axis with angle $\Phi$ (tilt angle). The light is linearly polarized in $\mathbf{x}$ $\mathbf{y}$ plane $\left(\mathbf{E}_{\omega}\right)$ with the angle of $\alpha$ from $\mathbf{x}$ direction and propagates in the $\mathbf{z}$ direction. SHG propagates in the emission plane, defined by $(\theta, \varphi)$.

Sheppard 2006; Chang and Deng 2010). A more complex but an actual situation that may exist is not further considered, for instance, the tilt-placed collagen fibrils which have a tilt angle of their axes from the plane perpendicular to the wave propagation direction. The in-depth study on such subject will be helpful for identifying the three-dimensional orientation of molecules in collagen fibrils, which will have potential interest in cancer and various diseases caused by modifications on the tissue/cellular structure. Therefore, in this paper, a theoretical model to deal with the tilt-placed collagen fibrils under a linearly polarized focused beam is to be established and the effects of the tilt-placed angle $\Phi$ on SHG radiation are to be investigated.

\section{Theory of SHG in tilt-placed fibrils under focused beam}

Under the two Cartesian coordinates (the fixed $\mathbf{x}-\mathbf{y}-\mathbf{z}$ and the transformed $\mathbf{x}^{\prime}-\mathbf{y}^{\prime}-\mathbf{z}^{\prime}$ coordinate systems) as showed in Fig. 1, the incident focused beam is assumed to propagate along $\mathbf{z}$ axis and has the electric field $\mathbf{E}_{\omega}$ polarized in $\mathbf{x}-\mathbf{y}$ plane with polarization angle $\alpha$ from $\mathbf{x}$ direction. The fibrils are hypothetically zero thickness and supposed to be composed of many infinitesimally small subunits (dipoles) aligned along $\mathbf{x}^{\prime}$ axis in the $\mathbf{x}-\mathbf{z}$ plane with an inclination angle $\Phi$ from $\mathbf{x}$ axis $\mathbf{x}^{\prime}$ thus is the symmetrical axis of the dipoles that conforms to cylindrical symmetry. The emission direction $\mathbf{r}$ of SHG deviates from $\mathbf{x}^{\prime}$ axis of angle $\psi$. The axis $\mathbf{z}$ and the emission direction $\mathbf{r}$ form the emission plane of SHG. The emitted SHG signals are confined within the solid angle defined by $(\theta, \varphi)$. SHG is supposed to be observed from a fixed distance $\mathbf{r}$ (Williams et al. 2005).

For a fibril laying along $\mathbf{x}$ axis, under the fixed coordinate system, the induced electric dipole moment (or polarization) per unit dipole concentration for SHG by a fundamental electric field $\mathbf{E}_{\omega}$ is (Moreaux et al. 2000)

$$
\mu_{2 \omega, i}=\frac{1}{2} E_{\omega}^{2} \sum \beta_{i j k} \varepsilon_{j} \varepsilon_{k}
$$

where $\beta$ is the first hyperpolarizability of dipoles and $i$ (also $\mathrm{j}$ and $\mathrm{k}$ ) refers to $\mathrm{x}, \mathrm{y}, \mathrm{z}$ respectively. $\varepsilon_{\mathrm{j}}$ and $\varepsilon_{\mathrm{k}}$ is the unit vector along the direction of $\mathrm{j}(\mathrm{x}, \mathrm{y}$ and $\mathrm{z}$ directions) and $\mathrm{k}(\mathrm{x}$, $y$ and $z$ directions), respectively. Accordingly, under the transformed coordinate of $\mathbf{x}^{\prime}-\mathbf{y}^{\prime}-\mathbf{z}^{\prime}$, it can be expressed as

$$
\mu_{2 \omega, i^{\prime}}=\frac{1}{2} \sum_{j^{\prime}, k^{\prime}}<\beta_{i^{\prime} j^{\prime} k^{\prime}}>E_{\omega, j^{\prime}} E_{\omega, k^{\prime}}
$$

The focused beam can be well approximated by a three-dimensional (3-D) Gaussian profile in $\mathbf{x}-\mathbf{y}-\mathbf{z}$ coordinate system as follows (Bjorkholm 1966; Deng et al. 2006):

$$
\begin{aligned}
& \vec{E}_{\omega}(x, y, z)=-i E_{\omega}^{(0)} \exp \left(-\frac{x^{2}+y^{2}}{w_{x y}{ }^{2}}-\frac{z^{2}}{w_{z}^{2}}+i \xi k_{\omega} z\right) \hat{\varepsilon} \\
& E_{\omega}^{(0)}=E_{\omega}(0,0,0)
\end{aligned}
$$

where $\mathbf{E}_{\omega}(\mathrm{x}, \mathrm{y}, \mathrm{z})$ is the complex amplitude of the driving field at the point $(\mathrm{x}, \mathrm{y}, \mathrm{z}), \boldsymbol{\varepsilon}$ represents its polarization direction, $\mathrm{k}_{\omega}=$ $2 \pi \mathrm{n}_{\omega} / \lambda_{\omega}$ is the wave vector in a specimen with refractive index of $\mathrm{n}_{\omega}$, $\xi$ is the wave vector reduction factor due to focusing that accounts for a reduction in axial momentum by conversion to lateral momentum components within the focus, $\mathrm{w}_{\mathrm{xy}}$ and $\mathrm{w}_{\mathrm{Z}}$ are the $1 / \mathrm{e}^{2}$ radii of the focal ellipse in the lateral and axial directions, respectively (Zipfel et al. 2003).

Based on the assumption of Cylindrical symmetry of fibrils and rule of Kleinman symmetry (Kleinman 1962; Mazely et al. 1987; Dailey et al. 2004; Rao et al. 2006; Chang et al. 2009), we have 7 non-zero but 2 independent elements of $\beta$, which are $\beta_{x^{\prime} x^{\prime} x^{\prime}} \beta_{x^{\prime} y^{\prime} y^{\prime}}=\beta_{x^{\prime} z^{\prime} z^{\prime}}=\beta_{y^{\prime} y^{\prime} x^{\prime}}=\beta_{z^{\prime} z^{\prime} x^{\prime}}=\beta_{y^{\prime} x^{\prime} y^{\prime}}=\beta_{z^{\prime} x^{\prime} z^{\prime}}$

In the case for the light linearly polarized in $\mathbf{x}-\mathbf{y}$ plane $\left(\mathbf{E}_{\omega}\right)$, there are three components of electrical field $\mathbf{E}_{\omega}$ in $\mathbf{x}^{\prime}, \mathbf{y}^{\prime}, \mathbf{z}^{\prime}$

$$
E_{x}=E_{\omega} \cos \alpha, E_{y}=E_{\omega} \sin \alpha, E_{z}=0
$$

Accordingly, three components of $\mathbf{E}_{\omega}$ in the transformed coordinate system $\mathbf{x}^{\prime}-\mathbf{y}^{\prime}-\mathbf{z}^{\prime}$ can be expressed as 


$$
\begin{aligned}
& E_{x^{\prime}}=E_{x} \cos \phi=E_{\omega} \cos \alpha \cos \phi \\
& E_{y^{\prime}}=E_{y}=E_{\omega} \sin \alpha \\
& E_{z^{\prime}}=E_{x} \sin \phi=E_{\omega} \cos \alpha \sin \phi
\end{aligned}
$$

Thus the dipole moments in three directions of $\mathbf{x}^{\prime}, \mathbf{y}^{\prime}, \mathbf{z}^{\prime}$ contributed by the electrical field $\mathbf{E}_{\omega}$ components, respectively, are following:

$$
\begin{aligned}
\mu_{2 \omega, x^{\prime}} & =\frac{1}{2} E_{\omega}^{2}\left(\beta_{x^{\prime} x^{\prime} x^{\prime}} \cos ^{2} \alpha \cos ^{2} \phi+\beta_{x^{\prime} y^{\prime} y^{\prime}} \sin ^{2} \alpha+\right. \\
& \left.+\beta_{x^{\prime} z^{\prime} z^{\prime}} \cos ^{2} \alpha \sin ^{2} \phi\right) \\
\mu_{2 \omega, y^{\prime}} & =\frac{1}{2} E_{\omega}^{2}\left(\beta_{y^{\prime} x^{\prime} y^{\prime}} \cos \alpha \cos \phi \sin \alpha+\right. \\
& \left.+\beta_{y^{\prime} y^{\prime} x^{\prime}} \cos \alpha \cos \phi \sin \alpha\right) \\
& =\frac{1}{2} E_{\omega}^{2}\left(\beta_{x^{\prime} y^{\prime} y^{\prime}} \sin 2 \alpha \cos \phi\right) \\
\mu_{2 \omega, z^{\prime}} & =\frac{1}{2} E_{\omega}^{2}\left(\beta_{z^{\prime} x^{\prime} z^{\prime}} \cos \alpha \cos \phi \cos \alpha \sin \phi+\right. \\
& \left.\beta_{z^{\prime} z^{\prime} x^{\prime}} \cos \alpha \cos \phi \cos \alpha \sin \phi\right) \\
& =\frac{1}{2} E_{\omega}^{2}\left(\beta_{x^{\prime} z^{\prime} z^{\prime}} \cos \alpha \sin 2 \phi\right)
\end{aligned}
$$

In order to clearly illustrate the contribution of hyperpolarizability tensor $\beta$ to dipole moment components along $\mathbf{x}^{\prime}, \mathbf{y}^{\prime}, \mathbf{z}^{\prime}$ directions, we keep three non-zero but two independent $\beta$ elements $\left(\beta_{x^{\prime} x^{\prime} x^{\prime}} \beta_{x^{\prime} y^{\prime} y^{\prime}}=\beta_{x^{\prime} z^{\prime}}\right)$ for our following discussion.

A matrix is applied to represent SHG dipole moment as

$$
\begin{gathered}
\vec{\mu}_{2 \omega}\left(x^{\prime}, y^{\prime}, z^{\prime}\right)=\left(\begin{array}{c}
\mu_{2 \omega, x^{\prime}} \\
\mu_{2 \omega, y^{\prime}} \\
\mu_{2 \omega, z^{\prime}}
\end{array}\right)=\frac{1}{2} \vec{E}_{\omega}^{2} \\
\left(\begin{array}{c}
\beta_{x^{\prime} x^{\prime} x^{\prime}} \cos ^{2} \alpha \cos ^{2} \phi+\beta_{x^{\prime} y^{\prime} y^{\prime}} \sin ^{2} \alpha+\beta_{x^{\prime} z^{\prime} z^{\prime}} \cos ^{2} \alpha \sin ^{2} \phi \\
\beta_{x^{\prime} y^{\prime} y^{\prime}} \sin 2 \alpha \cos \phi \\
\beta_{x^{\prime} z^{\prime} z^{\prime}} \cos ^{2} \alpha \sin 2 \phi \\
=\frac{1}{2} \vec{E}_{\omega}^{2} \beta
\end{array}\right)=
\end{gathered}
$$

We know that the electric field of SHG $\mathbf{E}_{2 \omega}$ induced by dipole moment obeys the following formula:

$$
\vec{E}_{2 \omega}(\psi)=\frac{\vec{\mu}_{2 \omega} \omega^{2}}{\pi \varepsilon_{0} c^{2} r} \sin (\psi) \exp \left(-i \vec{k}_{2 \omega} \cdot \vec{r}\right) \hat{\psi}
$$

where $\psi$ represents the angle between $\mathbf{x}^{\prime}$ axis and the emission direction $\mathbf{r}$ of SHG, $\sin \psi=\cos \theta \sin \Phi+\cos \varphi \sin \theta \cos \Phi$ illustrates the projection relationship between the direction of emission SHG dipole moment $\boldsymbol{\mu}_{2 \omega}\left(\mathrm{x}^{\prime}, \mathrm{y}^{\prime}, \mathrm{z}^{\prime}\right)$ and the excitation electric field $\mathbf{E}_{2 \omega}$ of SHG. We define $\eta=\omega^{2} / \pi \varepsilon_{0} c^{2}$, where $\varepsilon_{0}$ is the free-space permittivity, $c$ is the speed of light, $\omega$ is the frequency of fundamental beam.

Therefore, through integration, we get (Chang et al. 2009)

$$
\begin{aligned}
& \vec{E}_{2 \omega}(\theta, \varphi)=\left(\sqrt{\frac{\pi}{2}}\right)^{3} N_{V} w_{x y}^{2} w_{z} \frac{\eta}{r}(\cos \theta \sin \phi+ \\
& +\cos \varphi \sin \theta \cos \phi) \cdot \vec{\mu}_{2 \omega}^{(0)} \\
& \times \exp \left\{-\frac{k_{2 \omega}^{2}}{8}\left[w_{x y}^{2}(\sin \theta \cos \varphi \cos \phi)^{2}\right.\right. \\
& \left.\left.+w_{x y}^{2}(\sin \theta \sin \varphi)^{2}+w_{z}^{2}\left(\cos \theta \cos \phi-\xi^{\prime}\right)^{2}\right]\right\} \\
& =N \vec{E}_{2 \omega}^{(0)} A(\theta, \varphi) \\
& N=\left(\sqrt{\frac{\pi}{2}}\right)^{3} w_{x y}^{2} w_{z} N_{V} \\
& \vec{E}_{2 \omega}^{(0)}=\frac{\eta}{r}(\cos \theta \sin \phi+\cos \varphi \sin \theta \cos \phi) \cdot \vec{\mu}_{2 \omega}^{(0)} \\
& \vec{\mu}_{2 \omega}^{(0)}=\left(\begin{array}{l}
\mu_{2 \omega, x^{\prime}}^{(0)} \\
\mu_{2 \omega, y^{\prime}}^{(0)} \\
\mu_{2 \omega, z^{\prime}}^{(0)}
\end{array}\right)=\frac{1}{2} \vec{E}_{\omega}^{2}(0,0,0) \beta=\frac{1}{2} \vec{E}_{\omega}^{(0) 2} \beta \\
& A=\exp \left\{-\frac{k_{2 \omega}^{2}}{8}\left[w_{x y}^{2}(\sin \theta \cos \varphi \cos \phi)^{2}+\right.\right. \\
& \left.\left.+w_{x y}^{2}(\sin \theta \sin \varphi)^{2}+w_{z}^{2}\left(\cos \theta \cos \phi-\xi^{\prime}\right)^{2}\right]\right\}
\end{aligned}
$$

where $\mathrm{N}_{\mathrm{V}}$ represents the effective volume density of dipoles (Mertz and Moreaux 2001), and $\xi^{\prime}=\xi\left(\mathrm{n}_{\omega} / \mathrm{n}_{2 \omega}\right)$.

Electric field of SHG is normal to the emission direction r. Thus the radiated SHG with polarization components parallel (p) and perpendicular (s) to the emission plane can be derived as follows ( $p$ parallel to the emission plane and perpendicular to $\mathbf{r}, \mathrm{s}$ perpendicular to the emission plane):

$$
\begin{gathered}
\vec{E}_{2 \omega}(\theta, \varphi)=\left(\begin{array}{c}
E_{2 \omega, p}(\theta, \varphi) \\
E_{2 \omega, s}(\theta, \varphi)
\end{array}\right)=\frac{\eta}{r} \vec{M} \cdot \vec{\mu}_{2 \omega} \\
\vec{E}_{2 \omega}^{(0)}(\theta, \varphi)=\left(\begin{array}{c}
E_{2 \omega, p}^{(0)}(\theta, \varphi) \\
E_{2 \omega, s}^{(0)}(\theta, \varphi)
\end{array}\right)=\frac{\eta}{r} \vec{M} \cdot \vec{\mu}_{2 \omega}^{(0)}
\end{gathered}
$$


$\mathbf{M}$ is the projection matrix, which permutes coordinate between $\left(\mathbf{x}^{\prime}, \mathbf{y}^{\prime}, \mathbf{z}^{\prime}\right)$ and $(\theta, \varphi)$. It is defined by

$$
\vec{M}\left(\begin{array}{l}
\vec{\theta} \\
\vec{\varphi}
\end{array}\right)=\left(\begin{array}{ccc}
\cos \theta \cos \varphi \cos \phi & \cos \theta \sin \varphi & -\sin \theta \cos \phi \\
-\sin \varphi \cos \phi & \cos \varphi & 0
\end{array}\right)
$$

Then, for the amplitude of $\mathrm{E}_{2 \omega}$ in the $\theta$ direction $\left(\mathrm{E}_{2 \omega, \mathrm{p}}\right)$ and the amplitude of $\mathbf{E}_{2 \omega}$ in the $\varphi$ direction $\left(\mathrm{E}_{2 \omega, \mathrm{s}}\right)$, they are

$$
\begin{aligned}
E_{2 \omega, p}(\theta, \varphi)= & \frac{\eta N A(\theta, \varphi)}{2 r} E_{\omega}^{(0) 2} \\
& {\left[\cos \theta \cos \varphi \cos \phi \mu_{2 \omega, x^{\prime}}+\cos \theta \sin \varphi \mu_{2 \omega, y^{\prime}}\right.} \\
& \left.-\sin \theta \cos \phi \mu_{2 \omega, z^{\prime}}\right] \\
E_{2 \omega, s}(\theta, \varphi)= & \frac{\eta N A(\theta, \varphi)}{2 r} E_{\omega}^{(0) 2}\left[-\sin \varphi \cos \phi \mu_{2 \omega, x^{\prime}}\right. \\
& \left.+\cos \varphi \mu_{2 \omega, y^{\prime}}\right]
\end{aligned}
$$

The total SHG electric field can be expressed by

$$
E_{2 \omega}(\theta, \varphi)=\left[E_{2 \omega, p}^{2}(\theta, \varphi)+E_{2 \omega, s}^{2}(\theta, \varphi)\right]^{1 / 2}
$$

The power distribution of SHG can be determined by, $P_{2 \omega}(\theta, \varphi)=1 / 2 n_{2 \omega} \varepsilon_{0} c r^{2} E_{2 \omega}^{2}(\theta, \varphi)$, as a result, $P_{2 \omega}(\theta, \varphi)=\frac{1}{8} n_{2 \omega} \varepsilon_{0} c \eta^{2} A^{2} N^{2} E_{\omega}^{(0) 4}(\cos \theta \sin \phi+\cos \varphi \sin \theta \cos \phi)^{2}$

$$
\left(\begin{array}{c}
\beta_{x^{\prime} x^{\prime} x^{\prime}} \cos ^{2} \alpha \cos ^{2} \phi+\beta_{x^{\prime} y^{\prime} y^{\prime}} \sin ^{2} \alpha+\beta_{x^{\prime} z^{\prime} z^{\prime}} \cos ^{2} \alpha \sin ^{2} \phi \\
\beta_{x^{\prime} y^{\prime} y^{\prime}} \sin 2 \alpha \cos \phi \\
\beta_{x^{\prime} z^{\prime} z^{\prime}} \cos ^{2} \alpha \sin 2 \phi
\end{array}\right)^{2}
$$

SHG power of parallel and perpendicular components can be expressed as follows:

$$
\begin{aligned}
& P_{2 \omega, p}(\theta, \varphi)=\frac{1}{8} n_{2 \omega} \varepsilon_{0} c \eta^{2} N^{2} A^{2}(\theta, \varphi) E_{2 \omega, p}^{2} \\
& P_{2 \omega, s}(\theta, \varphi)=\frac{1}{8} n_{2 \omega} \varepsilon_{0} c \eta^{2} N^{2} A^{2}(\theta, \varphi) E_{2 \omega, s}^{2}
\end{aligned}
$$

As a result, SHG total power is

$$
P_{2 \omega}(\theta, \varphi)=P_{2 \omega, p}(\theta, \varphi)+P_{2 \omega, s}(\theta, \varphi)
$$

\section{Results}

In Fig. 2, we demonstrate the effects of the tilt angle $\Phi$ on overall strength of SHG electrical field at several

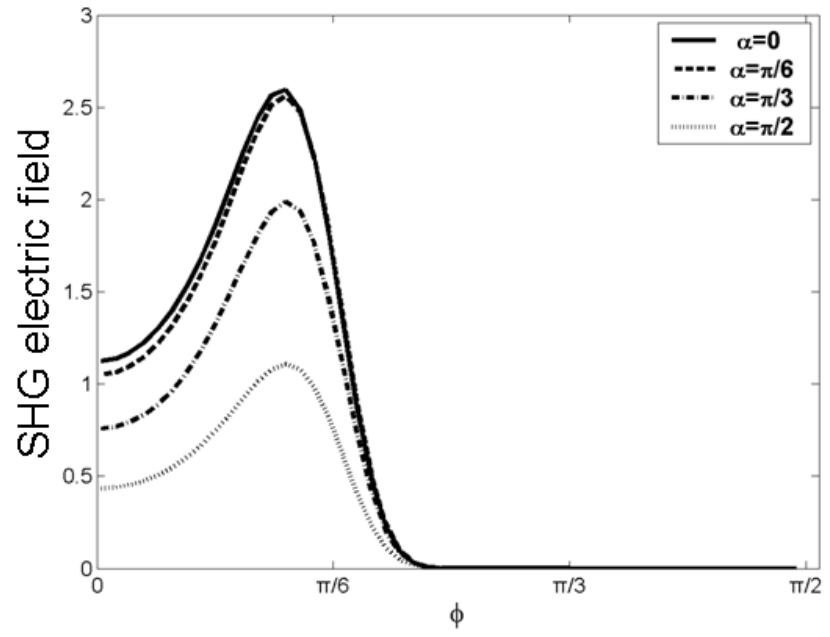

Figure 2. Effects of tilt angle $\Phi$ and polarization angle $\alpha$ on total SHG electric field $\left(\mathrm{E}_{2 \omega}\right)$.

particular polarization angles $\alpha=0^{\circ}, 30^{\circ}, 60^{\circ}, 90^{\circ}$. The overall strength of SHG electrical field is based on the solid angle integration of Eq. (17), in which we normalize

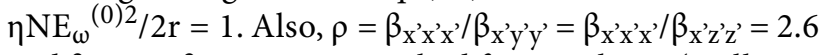
and $\beta_{x^{\prime} y^{\prime} y^{\prime}}=\beta_{x^{\prime} z^{\prime} z^{\prime}}=1$ are applied for simplicity (Williams et al. 2005; Odin et al. 2008). We can see from Fig. 2 that as the polarization angle $\alpha$ changes from $0^{\circ}$ to $90^{\circ}$ the overall emission strength of SHG electric field decreases but not uniformly. The far deviation of the polarization angle away from the $\mathbf{x}$ axis, the same interval of the polarization angle induces great reduction of the emission strength of SHG electrical field. Most importantly, we find that the influence of tilt angle $\Phi$ on overall strength of SHG electrical field does not increase or decrease linearly; on the contrary, the increase of tilt angle $\Phi$ first causes the increase and then decrease of the strength of the SHG electrical field. There is a maximum strength value of the SHG electrical field, which locates around $\Phi=27^{\circ}$. After $\Phi$ exceeds $45^{\circ}$, the strength of SHG electric field is almost undetectable.

Fig. 3 shows the distribution of SHG electric field parallel to the emission plane $\mathrm{E}_{2 \omega, \mathrm{p}}$ along the Cartesian coordinate axes induced by the dipole moment based on Eq. (16a). $\eta \mathrm{NE}_{\omega}{ }^{(0) 2} / 2 \mathrm{r}=1$ is applied here. The first, second and third column demonstrates $\mathrm{E}_{2 \omega, \mathrm{p}}$ under the cases of $\alpha=0^{\circ}, 45^{\circ}$ and $90^{\circ}$, respectively, as the variation of $\Phi=0^{\circ}$ to $\Phi=45^{\circ}$ with interval of $15^{\circ}$. The gray plane denotes the $\mathrm{z}=0$ level. Based on this plane, we can distinguish the field strengths associated with the backward SHG emission (b-SHG) or forward SHG emission (f-SHG). We can see that the tilt angle $\Phi$ of the fibrils axis has apparent influences on SHG electric field $E_{2 \omega, \mathrm{p}}$. When $\Phi$ exceeds $45^{\circ}$, the strength of SHG electric field is almost undetectable (thus no figures 

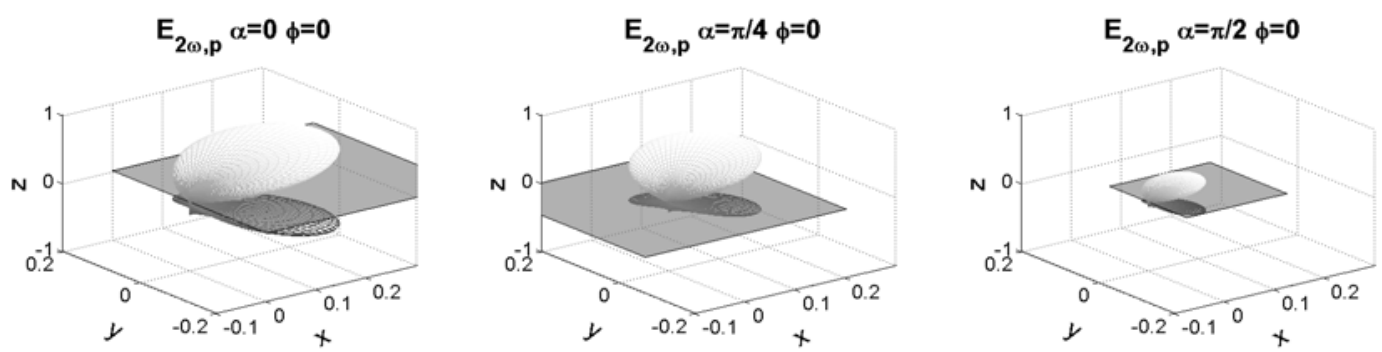

$E_{2 \omega, p} \alpha=0 \phi=\pi / 12$
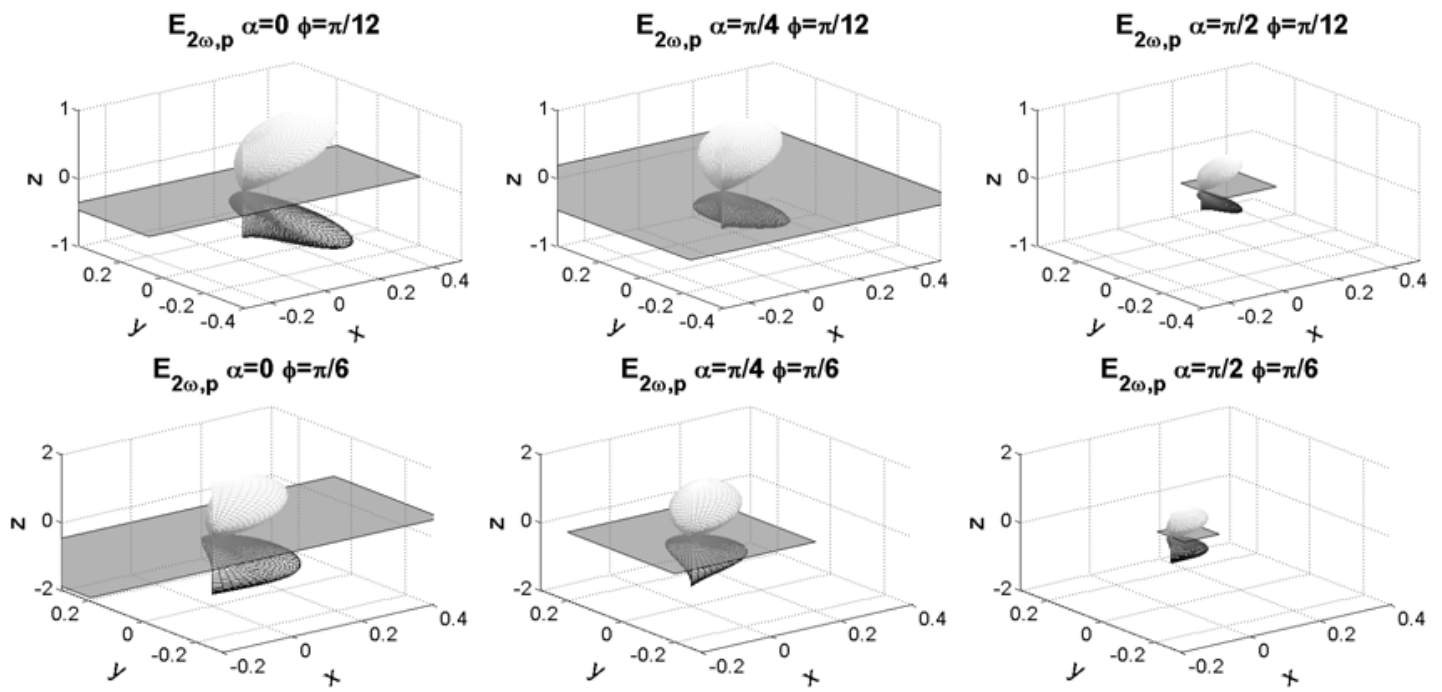

$E_{2 \omega, p} \alpha=0 \phi=\pi / 4$
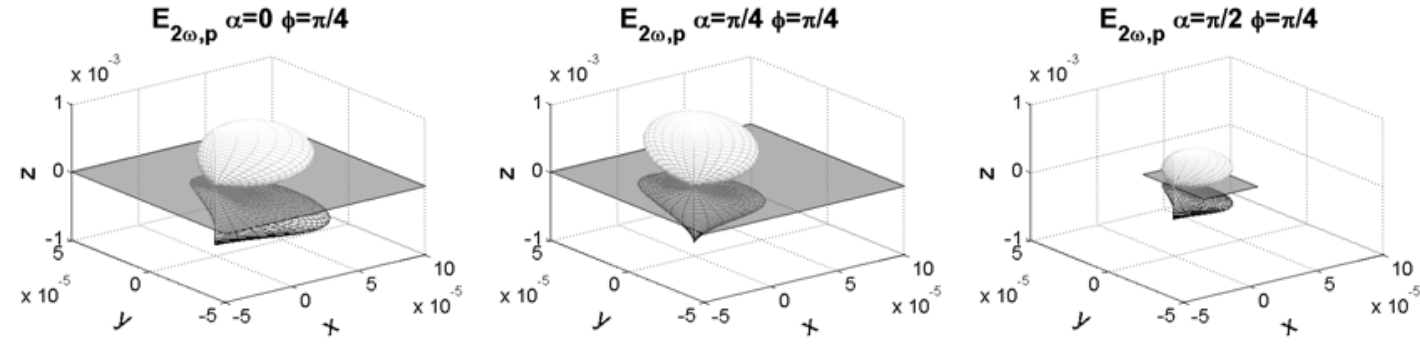

Figure 3. Effects of tilt angle $\Phi$ and polarization angle $\alpha$ on distribution of parallel component of SHG electric field $\left(\mathrm{E}_{2 \omega, \mathrm{p}}\right)$ along $\mathbf{x}-\mathbf{y}-\mathbf{z}$ axes.

are demonstrated here). Two symmetrical emission lobes of $E_{2 \omega, \mathrm{p}}$ which represent the b-SHG and f-SHG emission are clearly noticed in Fig. 3 . All $\mathrm{E}_{2 \omega, \mathrm{p}}$ distributes in the positive $\mathbf{x}$ direction. The polarization angle $\alpha$ and tilt angle $\Phi$ basically affect the strength size, but no the distribution shape of $\mathrm{E}_{2 \omega, \mathrm{p}}$.

Fig. 4 shows the distribution of SHG electric field perpendicular to the emission plane $\mathrm{E}_{2 \omega, \mathrm{s}}$ along the Cartesian coordinate axes induced by the dipole moment based on Eq. (16b). Also, the first, second and third column demonstrates $E_{2 \omega, s}$ under the cases of $\alpha=0^{\circ}, 45^{\circ}$ and $90^{\circ}$, respectively, as the variation of $\Phi=0^{\circ}$ to $\Phi=45^{\circ}$ with interval of $15^{\circ}$. Under the case of $E_{2 \omega, s}$, unlike two symmetrical f-SHG and b-SHG emission lobes in $\mathrm{E}_{2 \omega, \mathrm{p}}$, the pattern of SHG distribution of $E_{2 \omega, \mathrm{S}}$ resembles the hollow circular cone. Furthermore, in $\mathrm{E}_{2 \omega, \mathrm{s}}$, only b-SHG emission appears $\left(\alpha=0^{\circ}\right.$ and $\left.90^{\circ}\right)$. Two lobes still appear at the polarization angle of $\alpha=45^{\circ}$, but they are no longer symmetrical like that in $\mathrm{E}_{2 \omega, \mathrm{p}}$, b-SHG emission is predominant in this case.

Fig. 5 shows the distribution of SHG total electric field strength $\mathbf{E}_{2 \omega}$ along the Cartesian coordinate axes based on Eq. (17) under the cases of $\alpha=0^{\circ}$ (the first column), $\alpha=45^{\circ}$ (the second column), $\alpha=90^{\circ}$ (the third column), respectively, as the variation of tilt angle $\Phi=0$ to $\Phi=45^{\circ}$ with interval of $15^{\circ}$. We notice that when $\Phi$ is relative 

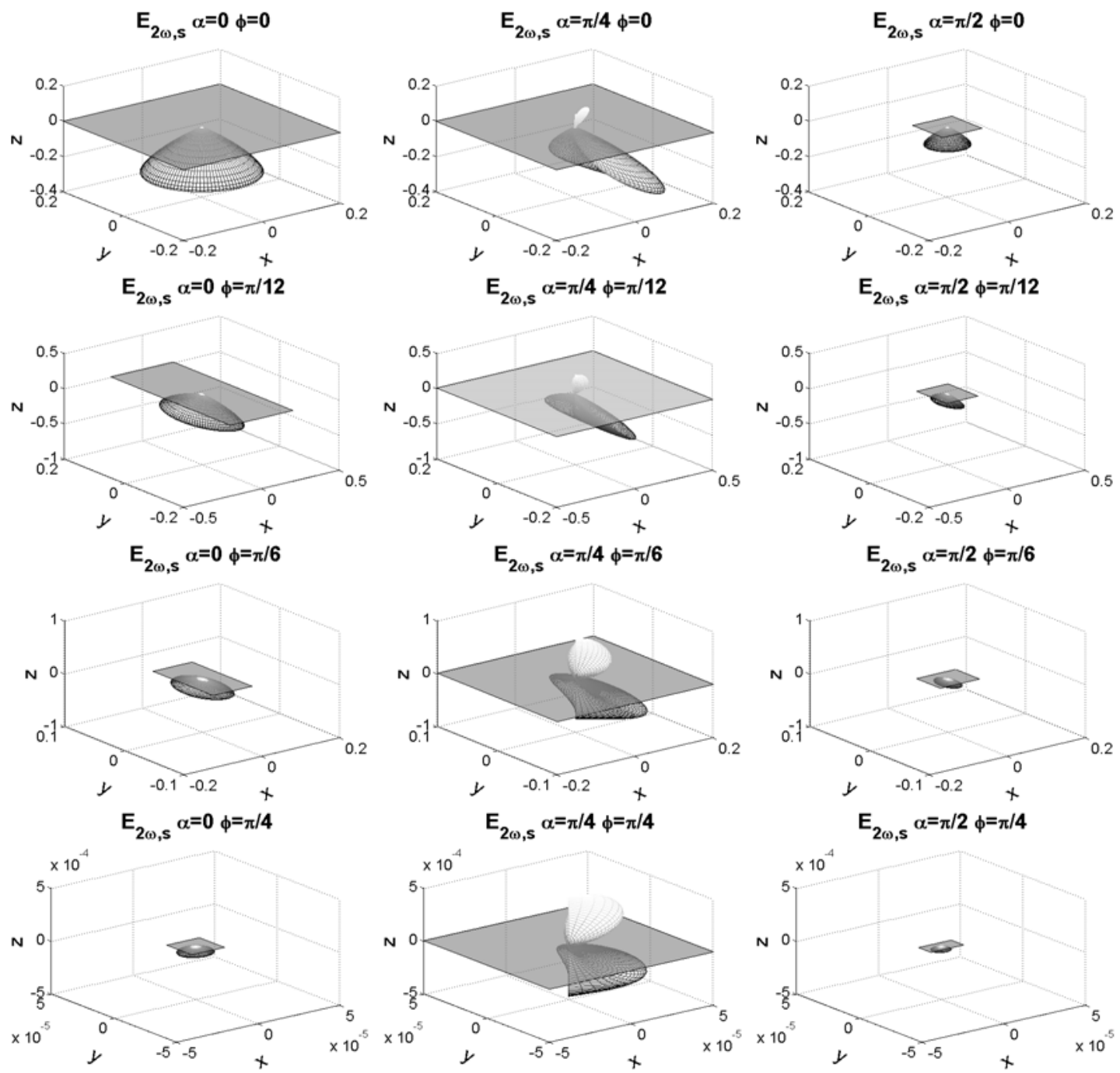

Figure 4. Effects of tilt angle $\Phi$ and polarization angle $\alpha$ on distribution of perpendicular component of SHG electric field $\left(\mathrm{E}_{2 \omega, \mathrm{s}}\right)$ along $\mathbf{x}-\mathbf{y}-\mathbf{z}$ axes.

smaller, such as $\Phi=0^{\circ}$ and $\Phi=15^{\circ}$, there are two emission lobes. However, when tilt angle $\Phi$ becomes bigger, such as $\Phi=30^{\circ}$ and $\Phi=45^{\circ}$, two apparently separated lobes gradually form to one integrated lobe. All the emission is in positive $\mathbf{z}$ direction, and no b-SHG distribution exists.

By solid angle integration of Eq. (20) and normalizing $\mathrm{n}_{2 \omega} \varepsilon_{0} \mathrm{c \eta}{ }^{2} \mathrm{~N}^{2} / 8=1$ as well as making an assumption of $\rho=$ $\beta_{x^{\prime} x^{\prime} x^{\prime}} / \beta_{x^{\prime} y^{\prime} y^{\prime}}=\beta_{x^{\prime} x^{\prime} x^{\prime}} / \beta_{x^{\prime} z^{\prime} z^{\prime}}=2.6\left(\beta_{x^{\prime} y^{\prime} y^{\prime}}=\beta_{x^{\prime} z^{\prime} z^{\prime}}=1\right)$, the total power of SHG as the variation of the tilt angle $\Phi$ has been investigated and be shown in Fig. 6. Simultaneously, the effect of polarization angels $\alpha=0^{\circ}, 30^{\circ}, 60^{\circ}, 90^{\circ}$ on total SHG emission power is also reflected in Fig. 6 . We notice that the peak SHG emission power always appears at a certain tile angle $\Phi$, here it is around $\Phi=27^{\circ}$ in spite of the polarization angles $\alpha$. In other words, the excitation polarization angle a would not affect the tile angle location of fibrils where it can emit the peak power. However, as the polarization angle a increases from $0^{\circ}$ to $90^{\circ}$, the total emission power of SHG decrease, but the degree of decrease is not even with the same interval of increase of polarization angle; the bigger of the polarization angle, the more decrease of the emission power. Also, at the range of $\Phi \geq 45^{\circ}$, there is no detectable SHG power.

Similarly, the distribution of parallel $\left(\mathrm{P}_{2 \omega, \mathrm{p}}\right)$ and perpendicular $\left(\mathrm{P}_{2 \omega, \mathrm{s}}\right)$ power components as the tile angle $\Phi$ and polarization angle $\alpha$ are demonstrated in Fig. 7 and Fig. 8 respectively based on Eq. (19a) and (19b). Here, 

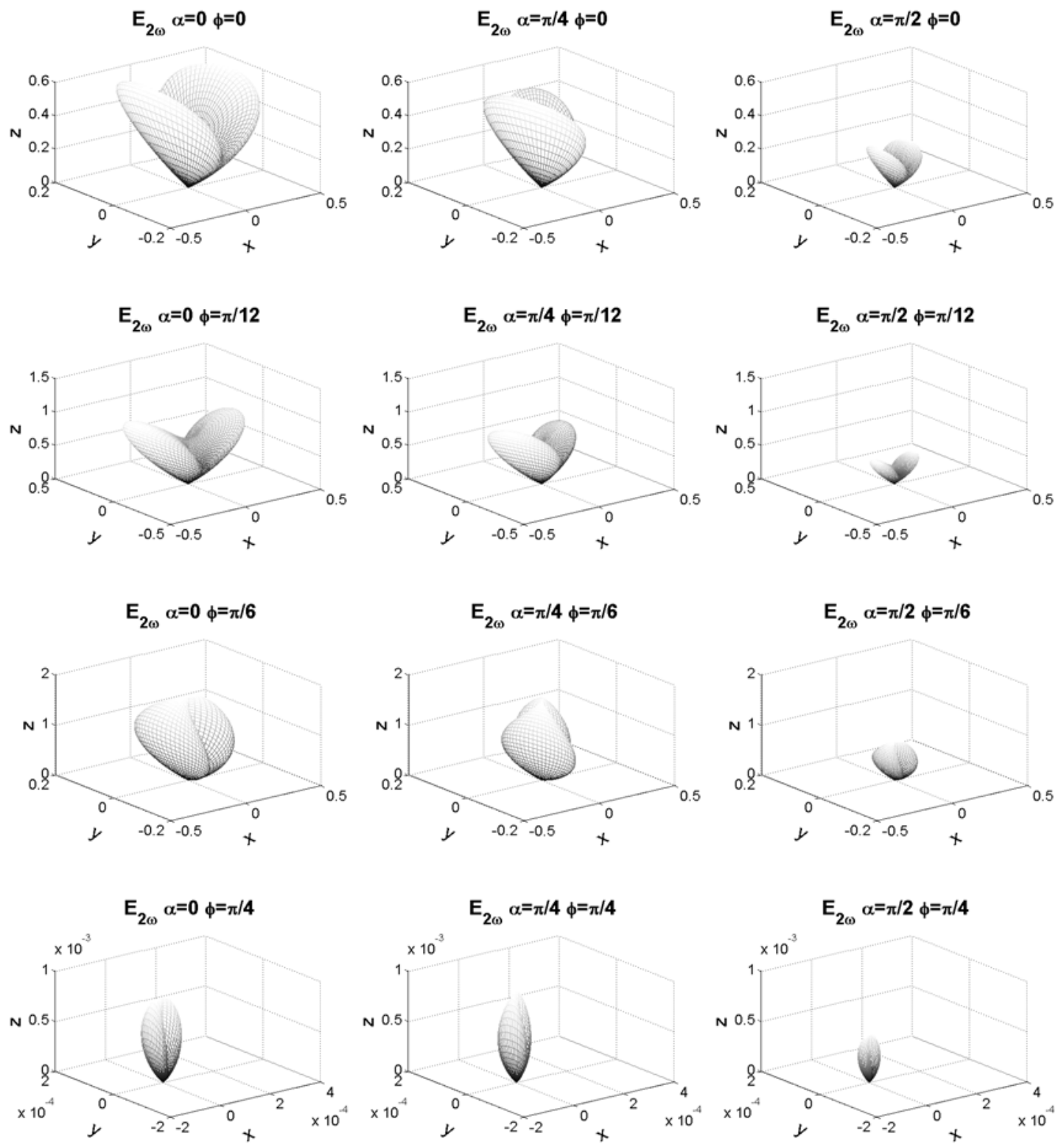

Figure 5. Effects of tilt angle $\Phi$ and polarization angle $\alpha$ on distribution of total SHG electric field $\left(E_{2 \omega}\right)$ along $\mathbf{x}-\mathbf{y}-\mathbf{z}$ axes.

$\mathrm{n}_{2 \omega} \varepsilon_{0} \mathrm{c \eta} \eta^{2} \mathrm{~N}^{2} / 8=1$ is applied. In Fig. 7, we notice that as the tilt angle $\Phi$ increases, two apparently detached emission lobes in $\mathrm{P}_{2 \omega, \mathrm{p}}$ progressively change to more closely touched one. The perpendicular component $\mathrm{P}_{2 \omega, \mathrm{s}}$ of $\mathrm{SHG}$ power shown in Fig. 8 declares hollow circular cone distribution along all demonstrated tile angles $\Phi$ at $\alpha=0^{\circ}$ and $\alpha=90^{\circ}$. However, the distribution of $\mathrm{P}_{2 \omega, \mathrm{s}}$ at $\alpha=45^{\circ}$ has obvious difference with those at $\alpha=0^{\circ}, \alpha=90^{\circ}$ and $P_{2 \omega, p}$ at $\alpha=$ $45^{\circ}$ as well. In $\mathrm{P}_{2 \omega, \mathrm{s}}$ at relative lower tile angles $\Phi=0^{\circ}$ and
$\Phi=15^{\circ}$, there is only one $\mathrm{f}-\mathrm{SHG}$ emission lobe, as the tile angle increases to $\Phi=30^{\circ}$ and $\Phi=45^{\circ}$, the one emission lobe gradually detach.

Accordingly, the distribution of SHG total power $\mathrm{P}_{2 \omega}$ along the Cartesian coordinate axes based on Eq. (20) has been demonstrated in Fig. 9. The distribution of SHG total power $\mathrm{P}_{2 \omega}$ as the polarization angle $\alpha$ and tile angle $\Phi$ is similar with the distribution pattern of SHG electric field $\mathrm{E}_{2 \omega}$. 


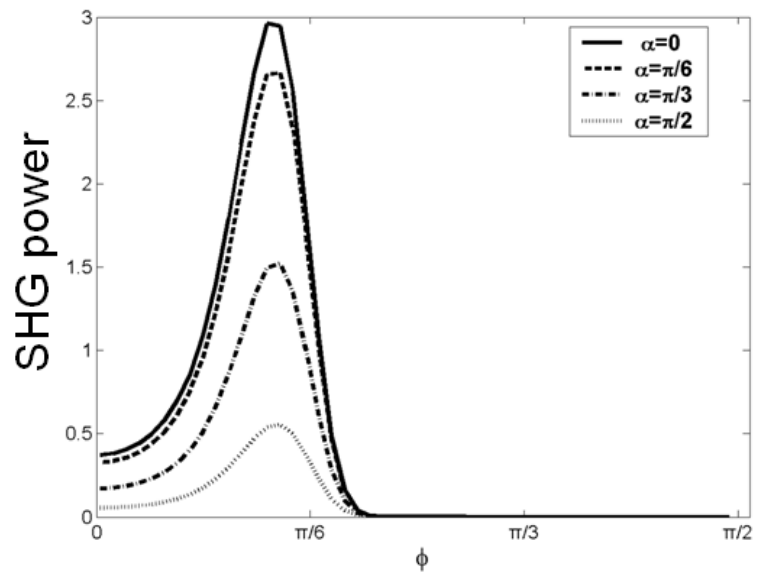

Figure 6. Effects of tilt angle $\Phi$ and polarization angle $\alpha$ on SHG total power $\left(\mathrm{P}_{2 \omega}\right)$.

\section{Discussion}

In this paper, microscopic SHG emission from tilt-placed collagen fibrils, e.i. the collagen fibrils being not in the same plane as the linear polarization direction of the focused excitation light, has been investigated. Based on this new model, the effects of the tilt angle $\Phi$ as well as the polarization angle $\alpha$ on total strength of electrical field and power (Fig. 2 and Fig. 6), the distribution of the total strength of electrical field $\mathrm{E}_{2 \omega}$ (Fig. 5) as well as its parallel ( $\left.\mathrm{E}_{2 \omega, \mathrm{p}}\right)$ (Fig. 3) and perpendicular components $\left(E_{2 \omega, s}\right)$ (Fig. 4) along $\mathbf{x}, \mathbf{y}, \mathbf{z}$ have been revealed. Also, the distribution of total power $\mathrm{P}_{2 \omega}$ (Fig. 9) and its parallel $\left(\mathrm{P}_{2 \omega, \mathrm{p}}\right)$ (Fig. 7) and perpendicular components $\left(\mathrm{P}_{2 \omega, \mathrm{s}}\right)$ (Fig. 8) along $\mathbf{x}, \mathbf{y}, \mathbf{z}$ have been investigated. We find that the tilt angle $\Phi$ does play an important role on SHG. The most apparent phenomenon is that the total strength
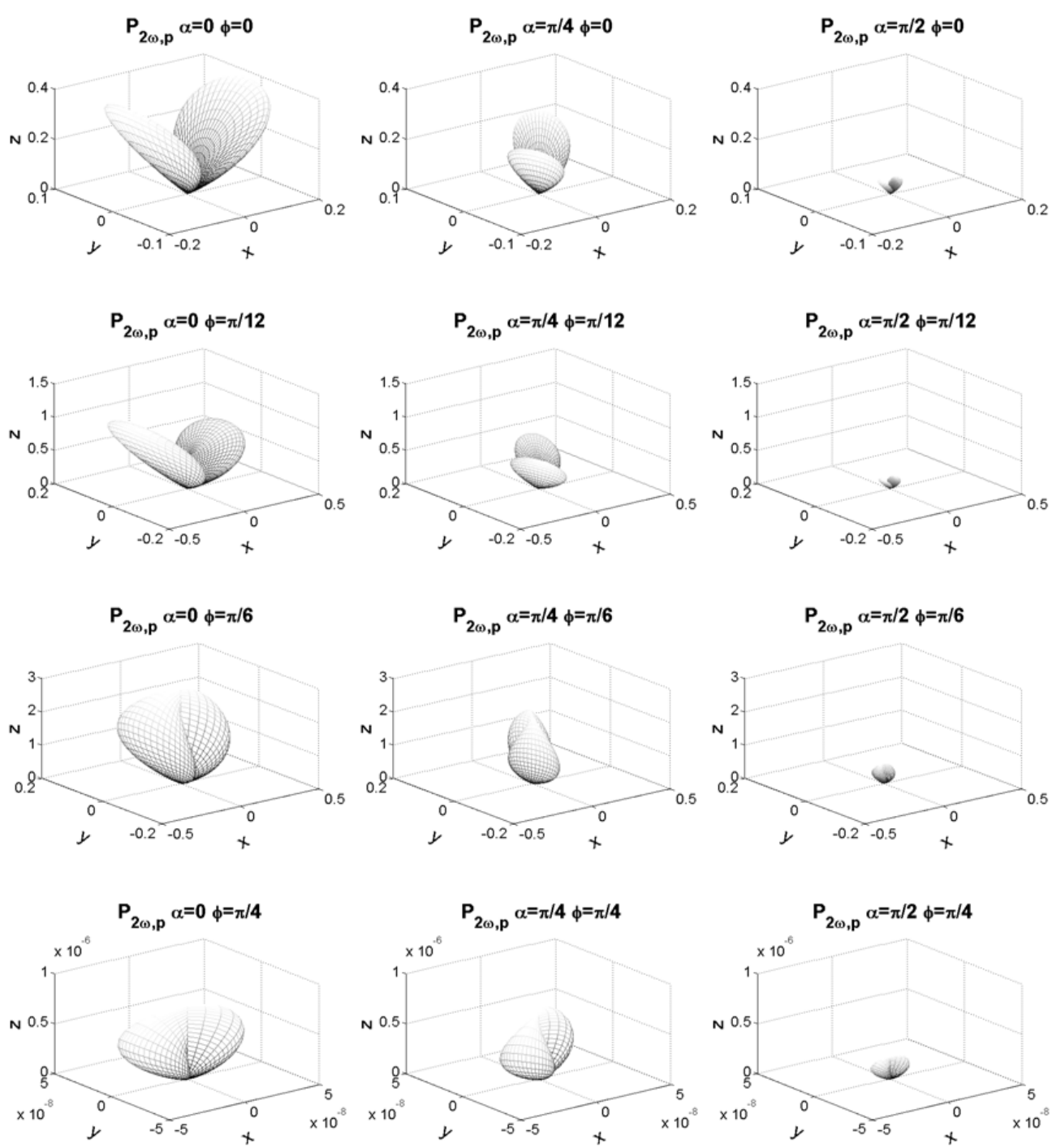

Figure 7. Effects of tilt angle $\Phi$ and polarization angle $\alpha$ on distribution of parallel component of SHG power $\left(\mathrm{P}_{2 \omega, \mathrm{p}}\right)$ along $\mathbf{x}-\mathbf{y}-\mathbf{z}$ axes. 

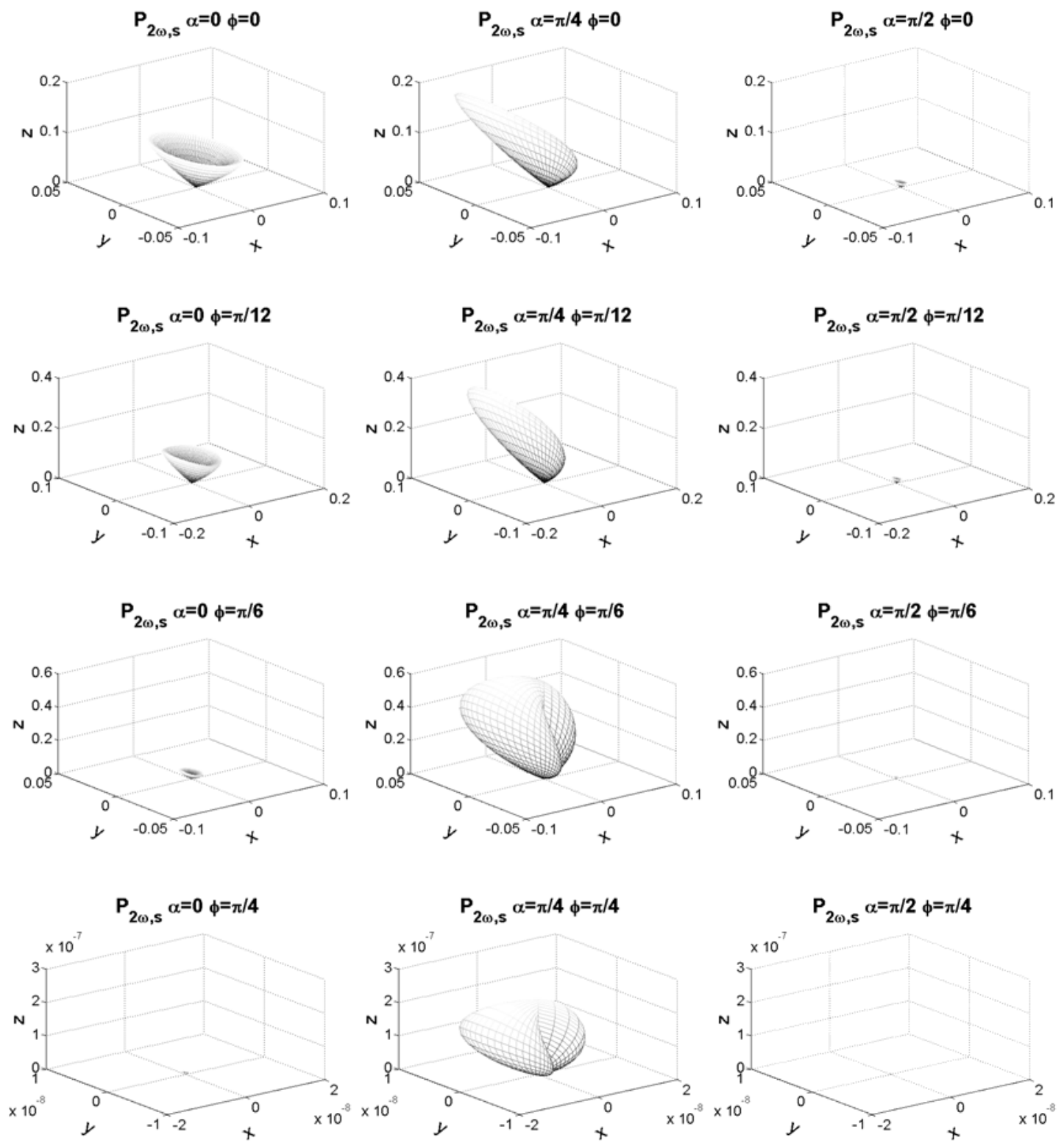

Figure 8. Effects of tilt angle $\Phi$ and polarization angle $\alpha$ on distribution of perpendicular component of SHG power $\left(\mathrm{P}_{2 \omega, \mathrm{s}}\right)$ along $\mathbf{x}-\mathbf{y}-\mathbf{z}$ axes.

of SHG electric field and power increase as the increase of tilt angle $\Phi$, and reach a maximum value when the fibril locates at a certain angel from the incident beam polarized plane, here it is $\Phi=27^{\circ}$. The continuous increase of fiber tilt angle $\Phi$ results in an abruptly decrease of the strength of SHG electric field and power. When tilt angle $\Phi$ exceeds around $45^{\circ}$, SHG signals become hardly detected. Also, the tilt angle $\Phi$ influences the distribution of the total strength of SHG electrical field and power along $\mathbf{x}, \mathbf{y}$ and $\mathbf{z}$ axes as well as their parallel and perpendicular components, such as the shape and the number of emission lobes. The polarization angle $\alpha$ has unevenly impact on the size of total strength of electrical field $\mathrm{E}_{2 \omega}$ and the power $\mathrm{P}_{2 \omega}$, the far deviation of polarization angle from $\mathbf{x}$ axis, the more evenly decrease of the total strength of electrical field $\mathrm{E}_{2 \omega}$ and the power $\mathrm{P}_{2 \omega}$. The polarization angle $\alpha=45^{\circ}$ has special influence on the distribution pattern of $\mathrm{E}_{2 \omega, \mathrm{s}}, \mathrm{E}_{2 \omega}, \mathrm{P}_{2 \omega, \mathrm{s}}$ and $\mathrm{P}_{2 \omega}$ compared to those of $\alpha=0^{\circ}$ and $90^{\circ}$.

Acknowledgements. The authors gratefully thank the National Natural Science Foundation of China (No. 30470495 and 30940020) for their supports. 

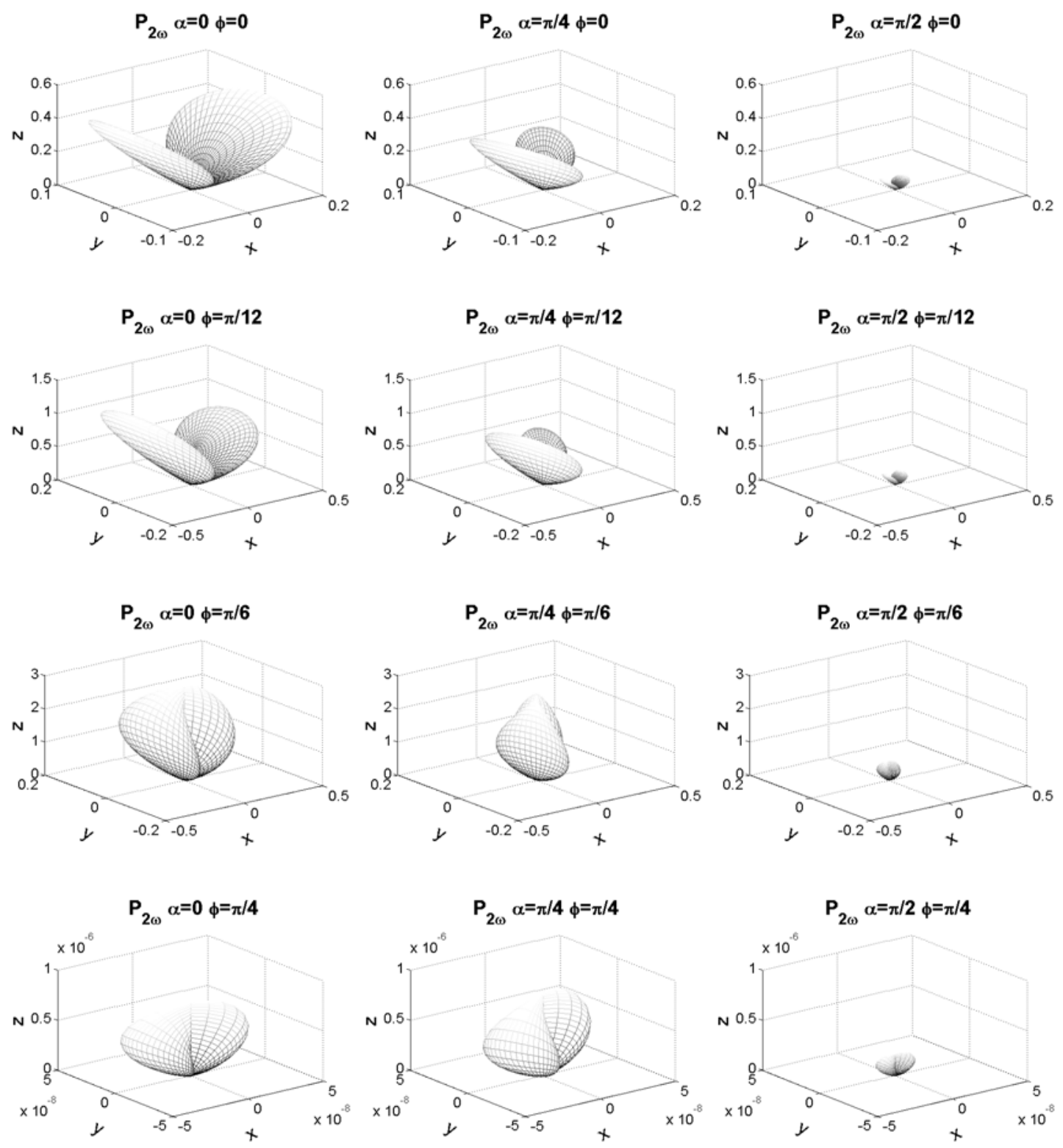

Figure 9. Effects of tilt angle $\Phi$ and polarization angle $\alpha$ on distribution of SHG total power $\left(P_{2 \omega}\right)$ along $\mathbf{x}-\mathbf{y}-\mathbf{z}$ axes.

\section{References}

Bjorkholm J. E. (1966): Optical бecond-harmonic generation using a focused gaussian laser beam. Phys. Rev. 142, 126 doi:10.1103/PhysRev.142.126

Brown E., McKee T., diTomaso E., Pluen A., Seed B., Boucher Y., Jain R. K. (2003): Dynamic imaging of collagen and its modulation in tumors in vivo using second-harmonic generation. Nat. Med. 9, 796-800 doi: $10.1038 / \mathrm{nm} 879$

Chang Y., Chen C. S., Chen J. X., Jin Y., Deng X. Y. (2009): Theoretical simulation study of linearly polarized light on microscopic second-harmonic generation in collagen type I. J. Biomed. Opt. 14

Chang Y., Deng X. Y. (2010): Characterization of excitation beam on second-harmonic generation in fibrillous type I collagen. J. Bio. Phys. 36, 365-383

Dailey C. A., Burke B. J., Simpson G. J. (2004): The general failure of Kleinman symmetry in practical nonlinear optical applications. Chem. Phys. Lett. 390, 8-13 doi:10.1016/j.cplett.2004.03.109

Deng X. Y., Wang X. J., Liu H. P., Zhuang Z. F., Guo Z. Y. (2006): Effect of distribution pattern of molecular dipoles on microscopic second-harmonic generation. Chin. Phys. Lett. 23, 629-632 
doi:10.1088/0256-307X/23/3/029

Helmchen F., Denk W. (2006): Deep tissue two-photon microscopy (vol 2, pg 932, 2005). Nat. Methods 3, 235-235 doi:10.1038/nmeth0306-235

Hompland T., Erikson A., Lindgren M., Lindmo T., Davies C. D. (2008): Second-harmonic generation in collagen as a potential cancer diagnostic parameter. J. Biomed. Opt. 13

Kleinman D. A. (1962): Nonlinear Dielectric Polarization in Optical Media. Phys. Rev. 126, 1977

doi:10.1103/PhysRev.126.1977

Kwan A. C., Dombeck D. A., Webb W. W. (2008): Polarized microtubule arrays in apical dendrites and axons. Proc. Natl. Acad. Sci. U. S. A. 105, 11370-11375 doi:10.1073/pnas.0805199105

Lamonier L., Bottcher-Luiz F., Pietro L., Andrade L., de Thomaz A. A., Machado C. L., Cesar C. L. (2010): Second harmonic generation in human ovarian neoplasias. Proc. SPIE, $75691 \mathrm{U}$ (75696 pp.)

Mazely T. L., Hetherington W. M. (1987): 2nd-order susceptibility tensors of partially ordered molecules on surfaces. J. Chem. Phys. 86, 3640-3647 doi:10.1063/1.451967

Mertz J., Moreaux L. (2001): Second-harmonic generation by focused excitation of inhomogeneously distributed scatterers. Opt. Commun. 196, 325-330 doi:10.1016/S0030-4018(01)01403-1

Moreaux L., Sandre O., Mertz J. (2000): Membrane imaging by second-harmonic generation microscopy. J. Opt. Soc. Am. B. 17, 1685-1694 doi:10.1364/JOSAB.17.001685

Odin C., Le Grand Y., Renault A., Gailhouste L., Baffet G. (2008) Orientation fields of nonlinear biological fibrils by second harmonic generation microscopy. J. Microsc.-Oxford 229, 32-38

Plotnikov S. V., Millard A. C., Campagnola P. J., Mohler W. A. (2006): Characterization of the myosin-based source for second-harmonic generation from muscle sarcomeres. Biophys. J. 90, 693-703 doi:10.1529/biophysj.105.071555

Psilodimitrakopoulos S., Petegnief V., Soria G., Amat-Roldan I., Artigas D., Planas A. M., Loza-Alvarez P. (2010): Polarization second harmonic generation (PSHG) imaging of neurons: estimating the effective orientation of the SHG source in axons. Proc. SPIE $75692 \mathrm{~W}$ doi:10.1117/12.841313
Psilodimitrakopoulos S., Santos S., Amat-Roldan I., Thayil A. K. N., Artigas D., Loza-Alvarez P. (2009): In vivo, pixel-resolution mapping of thick filaments' orientation in nonfibrilar muscle using polarization-sensitive second harmonic generation microscopy. J. Biomed. Opt. 14, 014001 doi: $10.1117 / 1.3059627$

Rao Y., Comstock M., Eisenthal K. B. (2006): Absolute orientation of molecules at interfaces. J. Phys. Chem. B 110, 1727-1732 doi:10.1021/jp055340r

Stoller P., Kim B. M., Rubenchik A. M., Reiser K. M., Da Silva L. B. (2002): Polarization-dependent optical second-harmonic imaging of a rat-tail tendon. J. Biomed. Opt. 7, 205-214 doi: $10.1117 / 1.1431967$

Stoller P. C., Reiser K. M., Celliers P. M., Rubenchik D. M. (2003): Effect of structural modification on second harmonic generation in collagen. Proc. SPIE 4963, 41-51 doi:10.1117/12.477998

Tiaho F., Recher G., Rouede D. (2007): Estimation of helical angles of myosin and collagen by second harmonic generation imaging microscopy. Opt. Express 15, 12286-12295 doi:10.1364/OE.15.012286

Williams R. M., Zipfel W. R., Webb W. W. (2005): Interpreting second-harmonic generation images of collagen I fibrils. Biophys. J. 88, 1377-1386 doi:10.1529/biophysj.104.047308

Yan F., Haifeng W., Riyi S., Ji-Xin C. (2007): Second harmonic and sum frequency generation imaging of fibrous astroglial filaments in ex vivo spinal tissues. Biophys. J., 3251-3259

Yew E. Y. S., Sheppard C. J. R. (2006): Effects of axial field components on second harmonic generation microscopy. Opt. Express 14, 1167-1174 doi:10.1364/OE.14.001167

Yew E. Y. S., Sheppard C. J. R. (2007): Second harmonic generation polarization microscopy with tightly focused linearly and radially polarized beams. Opt. Commun. 275, 453-457 doi:10.1016/j.optcom.2007.03.065

Zipfel W. R., Williams R. M., Webb W. W. (2003): Nonlinear magic: multiphoton microscopy in the biosciences. Nat. Biotechnol. 21, 1368-1376 doi:10.1038/nbt899

Received: September 15, 2010

Final version accepted: January 18, 2011 